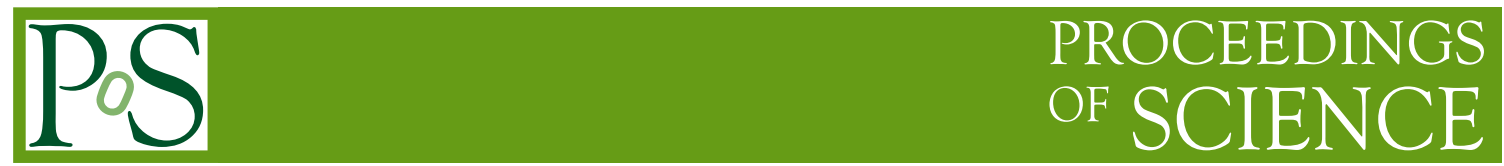

\title{
Looking the Universe from Deep Underground
}

\author{
Davide Trezzi (for the LUNA collaboration)* \\ Università degli Studi di Milano / INFN \\ E-mail: davide.trezzi@mi.infn.it
}

\begin{abstract}
The abundance of primordial light nuclei at the beginning of the Universe, during the so called Big Bang Nucleosynthesis era (BBN), can be estimated by means of Cosmology, Particle and Nuclear Physics. The first two give the environmental conditions and information about nucleons present at that time. The last one describes how those primordial nucleons fused themselves in order to produce the first light elements: helium, lithium and beryllium. The measurement of the nuclear fusion cross sections at energies of interest for BBN are thus very important. These cross sections are generally very low (from $\mathrm{pb}$ to $\mathrm{fb}$ and even smaller) and often impossible to be measured at laboratories on the Earth's surface due to the presence of cosmic rays. The only possibility is going deep underground where this kind of background is suppressed. Today, the only facility in the world able to do this is LUNA (Laboratory for Underground Nuclear Astrophysics), located in the LNGS (Laboratori Nazionali del Gran Sasso, Italy) laboratory. Thanks to the background suppression provided by about 1400 meters of rock and to the high current $400 \mathrm{kV}$ accelerator, LUNA is able to investigate cross sections at energies of astrophysical interest $(<400 \mathrm{keV}$ in the lab system), giving us the unique possibility to reproduce reactions that, in the past, produced the primordial elements now visible in astrophysical environments such as metal poor stars. In this paper an overview on nuclear astrophysics and BBN will be given, focusing on the latest measurements obtained at LUNA.
\end{abstract}

53rd International Winter Meeting on Nuclear Physics

26-30 January 2015

Bormio, Italy

${ }^{*}$ Speaker. 


\section{The Big Bang Nucleosynthesis}

The simplest cosmological model that better describes the evolution of our Universe is the Lambda Cold Dark Matter ( $\Lambda \mathrm{CDM})$ model. This one is based on three important experimental evidences: the cosmic expansion, the Cosmic Microwave Background (CMB) radiation and the Big Bang Nucleosynthesis (BBN). The first one was observed by Edwin Hubble and, in the $\Lambda \mathrm{CDM}$ model, it is included in the Fridman-Lemaitre equation. The CMB radiation, discovered by Penzias and Wilson, has been recently measured by the Planck mission [1]. It represents the thermal radiation left over from the Big Bang i.e. the starting point of the history of Universe. Assuming the $\Lambda \mathrm{CDM}$ model, this should be a self-consistent theoretical framework only if the CMB radiation power spectrum and the expansion of the Universe are in agreement each other. Consequentially, using expansion and $\mathrm{CMB}$ radiation measurements in order to fix the cosmological parameters of the $\Lambda \mathrm{CDM}$ model, it is possible to estimate the primordial abundances using BBN. This theory is able to predicts the primordial abundances of the "light cosmological nuclei" such as hydrogen, helium, lithium and beryllium that have been produced during the first 20 minutes after the Big Bang. However BBN requests other inputs: the temperature and baryon density of the Universe, the starting number of neutrons respect to protons ( $\mathrm{n} / \mathrm{p}$ ratio) and the cross section of all the nuclear reactions involved in the production and destruction of the primordial nuclei. The temperature $T$ and the baryon density $\left(\rho_{b}\right)$ of the Universe depend on time and they can be calculated using the Fridman-Lemaître equation. Assuming a flat Universe dominated by radiation we have:

$$
T(t)=\left[\frac{3 c^{2}}{32 \pi G a\left(1+\left(\frac{4}{11}\right)^{4 / 3} \frac{7}{8} N_{v}\right)}\right]^{1 / 4} \sqrt{\frac{1}{t}}
$$

where $c$ is the speed of light, $G$ is the gravitational constant, $a$ the Stefan-Boltzmann constant and $N_{v}$ is the number of neutrino families. In the $\Lambda$ CDM model a value of $N_{v}=3.04$ is assumed [2]. Using the same formalism it's possible to obtain the time evolution of the Universe density:

$$
\Omega_{b}(t)=\Omega_{b}(\text { now })\left[\frac{T(t)}{T(\text { now })}\right]^{3}
$$

where $\Omega_{b}=\frac{\rho_{b}}{\rho_{c}}$ is the baryon density expressed in critical density unity where $\rho_{c}=3 H_{0}^{2} / 8 \pi G$ with $H_{0}$ the today Hubble constant value. The neutron to proton ratio could be calculated using the Standard Model of Particle Physics and, at the freezeout temperature $\left(T=10^{10} \mathrm{~K}\right)$ a value $\mathrm{n} / \mathrm{p} \simeq$ $1 / 6$ is assumed [3]. This temperature is related to the period when the equilibrium between neutron production and destruction thought weak and electromagnetic interactions broke. After that time the $\mathrm{n} / \mathrm{p}$ ratio changed due to the free neutron decay:

$$
\left(\frac{n}{p}\right)=\left(\frac{n}{p}\right)_{\text {freezeout }} \exp \left(-\frac{t}{\tau}\right)
$$

where $\tau$ is the neutron lifetime [3]. When the temperature of the Universe become lower than the binding energy of deuteron nucleus $(2.2 \mathrm{MeV})$, the proton capture reaction $\mathrm{n}(\mathrm{p}, \gamma)^{2} \mathrm{H}$ took place 


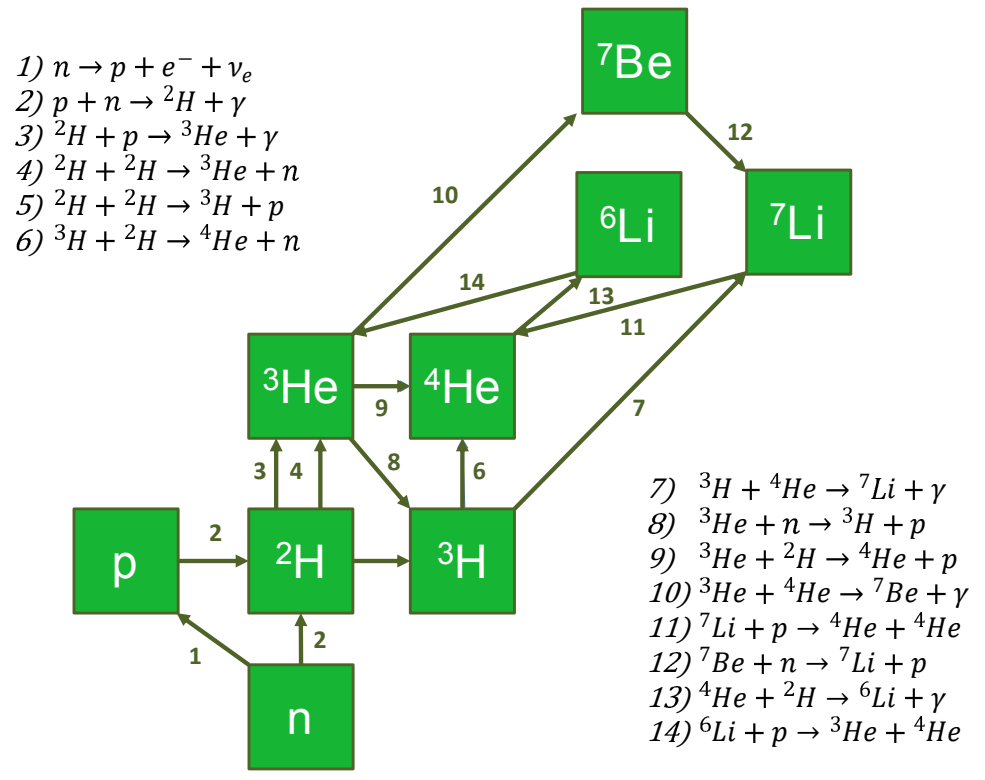

Figure 1: Nuclear reactions involved during the BBN era.

and the first nuclei in the Universe had been created. However, in order to have a consistent amount of deuterium, it was necessary to wait when the Universe temperature decreased to $10^{9} \mathrm{~K}$ because, given to the high radiation density at that time, the newcomer deuterium nuclei were destroyed through photo-disintegration ${ }^{2} \mathrm{H}(\gamma, \mathrm{p}) \mathrm{n}$. This condition is the so called deuterium bottleneck and fixes the starting point of BBN and corresponds to about three minutes and half after the Big Bang. Overpassed the deuterium bottleneck, protons, neutrons and deuterons started to produce heavy isotopes such as ${ }^{3} \mathrm{H},{ }^{3} \mathrm{He},{ }^{4} \mathrm{He},{ }^{6} \mathrm{Li},{ }^{7} \mathrm{Li}$ and ${ }^{7} \mathrm{Be}$ through the reaction network reported in figure 1.

The production processes continued for about five hours and half. After that period, the Universe became so cold that no nuclear fusion reaction could take place and the survived free neutrons decayed into protons. At the end of the BBN, the chemical composition of the Universe was completely determined and no new isotopes had been produced until the first stars had been created, about 500 million years after the Big Bang.

\section{Nuclear Astrophysics Deep Underground}

In order to calculate the abundances of the primordial elements produced during the BBN era, the knowledge of the related processes cross sections is needed. These fusion reactions occur in a very well defined energy range called Gamow window [4]. This one arises from the convolution of the Maxwell-Boltzmann distribution of nuclei in non-degenerate and non-relativistic stellar plasma with the tunneling probability through the Coulomb barrier between the interacting nuclei. The resulting distribution shows a maximum, the so called Gamow peak at energy $E_{0}=1.22\left(Z_{1}^{2} Z_{2}^{2} \mu T_{6}^{2}\right)^{1 / 3}$ where $Z_{1}$ and $Z_{2}$ are the integral charges of the interacting nuclei, $\mu$ is the reduced mass of the colliding system expressed in a.m.u. and $T_{6}$ the temperature in million Kelvin. For what concern the Big Bang Nucleosynthesis the Gamow peak varies between a few 
tents $\mathrm{keV}$ to hundreds $\mathrm{keV}$. Consequentially, the nuclear fusion reaction cross sections drop to very low values down to the femto-barn level. It follows that a direct investigation of thermonuclear reaction in the energy range of interest for BBN is often beyond technical capabilities given that the signal-to-noise ratio is severely dominated by different unwanted backgrounds. For what concerns fusion reactions, excluding a possible beam induced background and environmental radiation, the main contribution to the background comes from cosmic rays interaction both with detectors and surrounding materials. In a Earth's surface laboratory, the reaction cross sections are thus measured at high energies and extrapolated to the BBN energy range. The extrapolation procedure is usual carried out on the so-called astrophysical S-factor [4] defined by:

$$
S(E)=E \sigma(E) \exp (2 \pi \eta)
$$

where $\sigma(E)$ is the cross section, $E$ the centre-of-mass (cms) energy in $\mathrm{keV}$ and $\eta$ the Sommerfeld parameter given by:

$$
\eta=\frac{31.29}{2 \pi} Z_{1} Z_{2}\left(\frac{\mu}{E}\right)^{1 / 2}
$$

The astrophysical S-factor contains all the strictly nuclear effects and for nonresonant reactions this is a smoothly varying function of energy and it varies less rapidly with energy than the cross section. However the presence of unknown narrow resonances in or near the Gamow window, the tails of broad resonances and/or sub-threshold states, as well as a change in the reaction mechanism at ultra-low energies could affect on the cross section making the extrapolation procedure extremely dangerous. The only possibility to directly access to BBN energies is to reduce the cosmic background. This can be achieved by carrying out the cross section measurement in a deep underground laboratory such as the Laboratori Nazionali del Gran Sasso (LNGS). Here, a reduction of a factor $10^{6}$ in muon, $10^{3}$ in neutron and $10^{4}-10^{5}$ in gamma flux is provided. These optimal condition can be improved by the use of radiation shields, more efficient underground thanks to the secondary radiation reduction. Since 1998, the Laboratory for Underground Nuclear Astrophysics (LUNA) experiment is installed at the LNGS, devoted to cross section measurements at the astrophysical energies of interest [5,6]. Core of the LUNA experiment is the $400 \mathrm{kV}$ electrostatic accelerator [7]. The high voltage is generated by an Inline-Cockcroft-Walton power supply capable to provide an high current proton, alpha and ${ }^{3} \mathrm{He}$ beam at the maximum energy of $400 \mathrm{keV}$ with precise absolute energy $( \pm 0.3 \mathrm{keV})$, low spread $(<0.1 \mathrm{keV})$ and long-term stability $(5 \mathrm{eV} / \mathrm{h})$. The beam is sheared by two lines devoted respectively to gas and solid targets (see figure 2).

In the first case (beam line \#1), the electrostatically guided particles impinged on a constant pressure gas target. The beam current is measured with a constant-gradient calorimeter. This system is characterized by two sides: one kept at $70^{\circ} \mathrm{C}$ and the other cooled to $0^{\circ} \mathrm{C}$. The temperature difference is maintained constant. When the beam hits the calorimeter, less power is necessary to keep the gradient constant. Starting from the power difference between beam-on and beam-off conditions it's possible to calculate the beam current. The gas target is windowless in order to guarantee the optimum beam energy resolution provided by the accelerator. Because of the absence of any physical barrier between the gas target (pressure up to $1 \mathrm{mbar}$ ) and the ultra high vacuum in the accelerator $\left(10^{-7}\right.$ mbar), a differentially pumped system is necessary. The gas pressure is controlled by an analog feedback system and logged permanently by a Labview application. In 


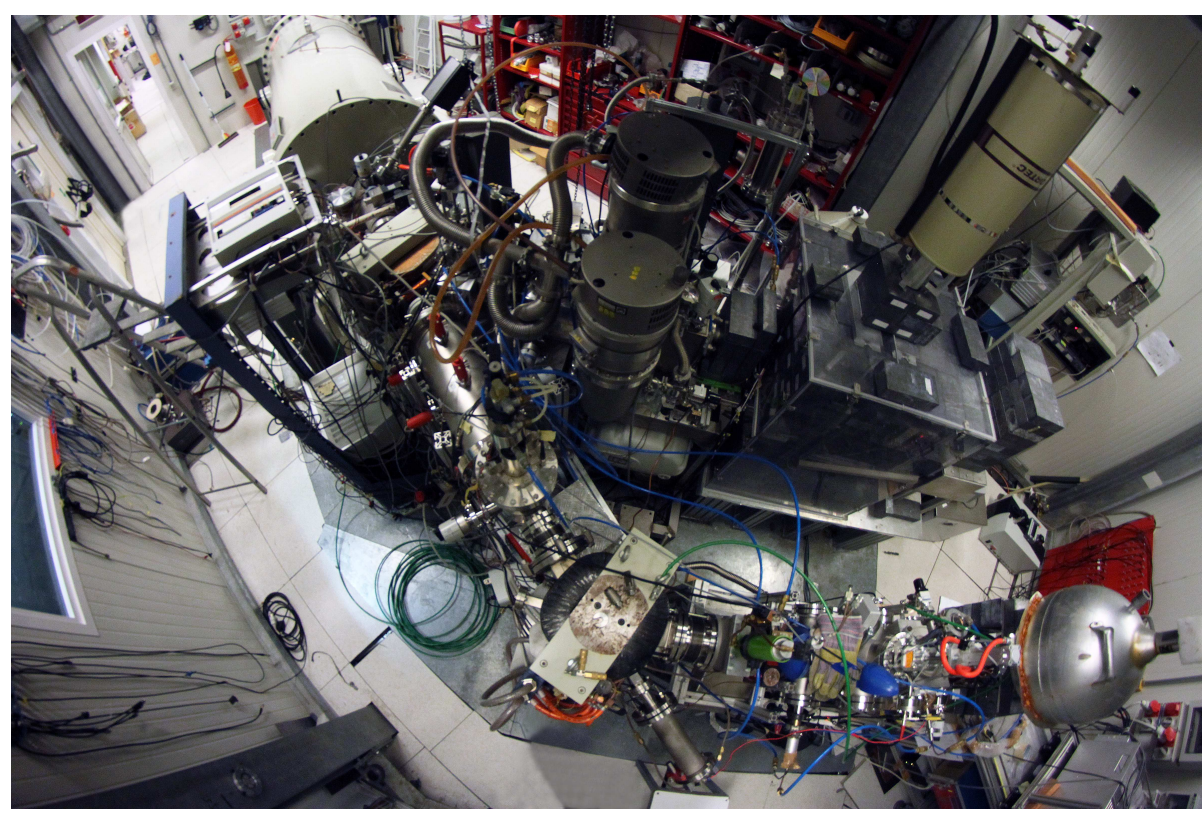

Figure 2: The LUNA (Laboratory for Underground Nuclear Astrophysics) setup. A technical description of the apparatus is reported in $[5,6]$.

order to shield the system from environmental radioactivity and residual cosmic background, a lead castle and an anti-radon box could be mounted around the gas target. The reaction gamma rays are thus detected by an High Purity Germanium, NaI or BGO detectors. The second beam line (beam line \#2) consists in a system of quadrupoles able to focus the beam on a water cooled solid target. A cooling trap is mounted in order to avoid a possible contamination of the target surface. The reaction gamma rays are detected by an High Purity Germanium or BGO detector. For charged particle reactions a single or an array of silicon detectors can be installed. The beam current is measured using a Faraday cup provided with an electron suppression system. In both lines a set of Faraday cups and aperture permit to perform a good beam collimation. The LUNA scientific activity in the last twenty years was mainly devoted to the study of fusion reactions involved in stars (sun-like stars, $\mathrm{RGB}, \ldots$ ) and novae [5,6]. However, recently the ${ }^{2} \mathrm{H}(\alpha, \gamma){ }^{6} \mathrm{Li}$ reaction has been investigated in the BBN energy range [8] and new key reaction from the cosmological point of view will be investigated in a close future like the ${ }^{2} \mathrm{H}(\mathrm{p}, \gamma){ }^{3} \mathrm{He}$ [9] and ${ }^{3} \mathrm{He}(\alpha, \gamma){ }^{7} \mathrm{Be}$ [10]. This last one has been already studied at LUNA with the $400 \mathrm{keV}$ machine [11] but new data at higher energies could be performed with the new 3.5 MeV LUNA-MV machine (details in [10]).

\section{Big Bang Nucleosynthesis at LUNA}

In the follow a short description of the fusion reactions investigated at LUNA related to BBN are given. For what concerns the ${ }^{2} \mathrm{H}(\mathrm{p}, \gamma)^{3} \mathrm{He}$, it will scheduled to be measured at LUNA at the end of 2015 and at the beginning of 2016. In this paper a short review on the experimental data present in literature and a brief discussion on recent LUNA results are reported. 


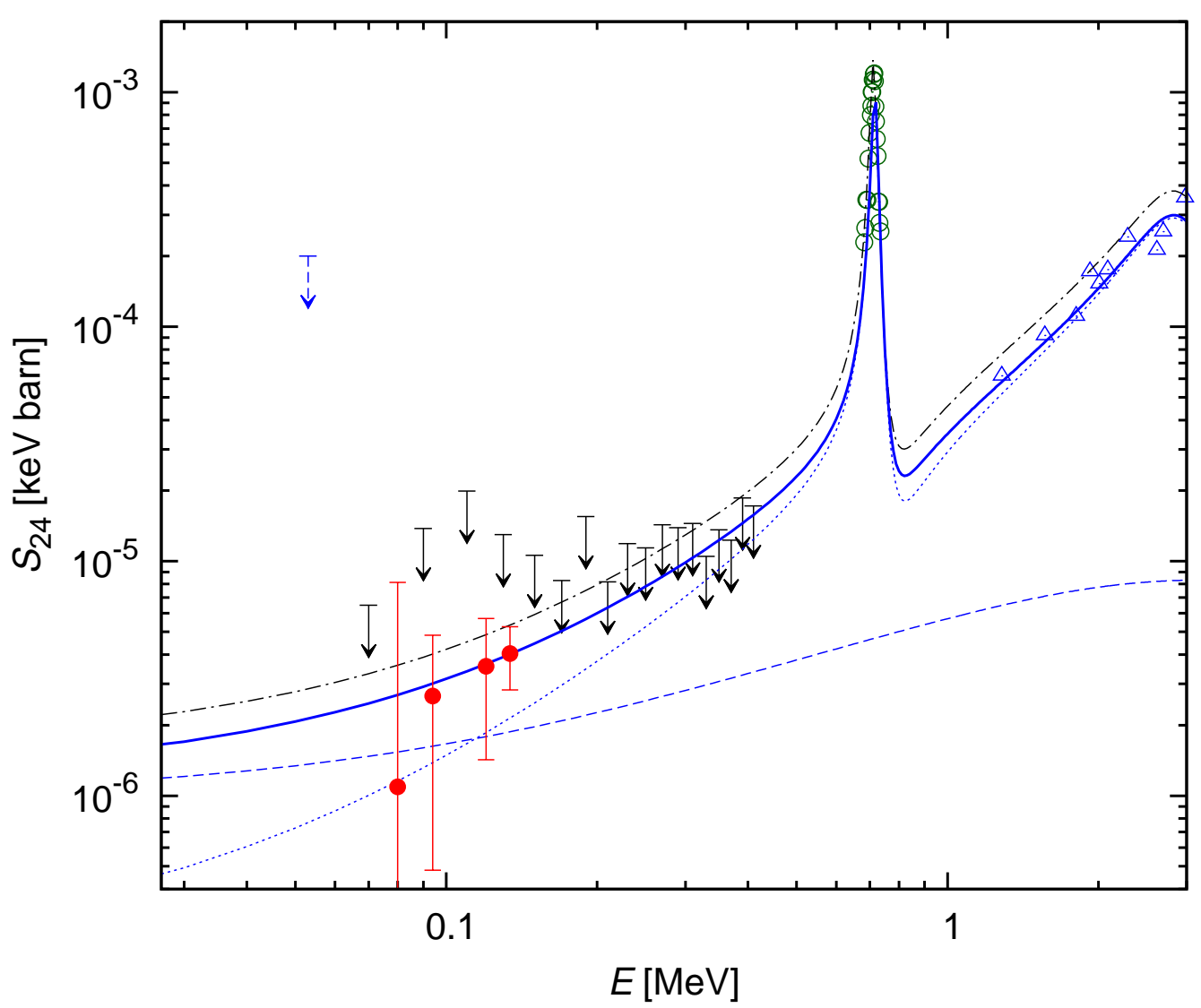

Figure 3: The ${ }^{2} \mathrm{H}(\alpha, \gamma)^{6} \mathrm{Li}$ astrophysical S-factor. Red dots rapresents the LUNA data points. Previous measurements are also reported (blue triangles [15], green circle [16], black arrows [17] (upper limits), blue dashed arrow [18] (upper limits). The blue full line is the theoretical Mukhamedzhanov E1+E2 cross section curve [19] and in dashed the E1 (long) and E2 (short) single component. We report also the Hammache teoretical cross section curve (black dot dashed) [20]

\subsection{The ${ }^{2} \mathbf{H}(\alpha, \gamma)^{6} \mathrm{Li}$ reaction}

${ }^{6} \mathrm{Li}$ is one of the heaviest isotopes produced during the BBN. Its abundance is dominated by two nuclear reaction responsible of the production and destruction of this fragile nucleus: the ${ }^{2} \mathrm{H}(\alpha, \gamma){ }^{6} \mathrm{Li}$ and the ${ }^{6} \mathrm{Li}(\mathrm{p}, \alpha)^{3} \mathrm{He}$ [12]. However, while the latter reaction rate is well known, the former has been only recently measured by LUNA in the BBN energy range [8] due to its very low cross section. For this measurement, a gas target filled with deuterium has been used [13]. If this setup guarantees a long term target stability, a huge beam induced background has been observed. This is due to high energy scattered deuterons by the $\alpha$-beam able to produce neutrons trough the ${ }^{2} \mathrm{H}\left({ }^{2} \mathrm{H}, \mathrm{n}\right)^{3} \mathrm{He}$ reaction [13, 14]. This fast neutrons interacting with the surrounding materials produced a (n,n' $\gamma$ ) gamma background, decreasing the signal to noise value to about one hundredth.

The situation of the ${ }^{2} \mathrm{H}(\alpha, \gamma)^{6} \mathrm{Li}$ before and after LUNA is shown in figure 3.

LUNA data excludes a possible low energy resonant contribution in agreement with the Mukam- 


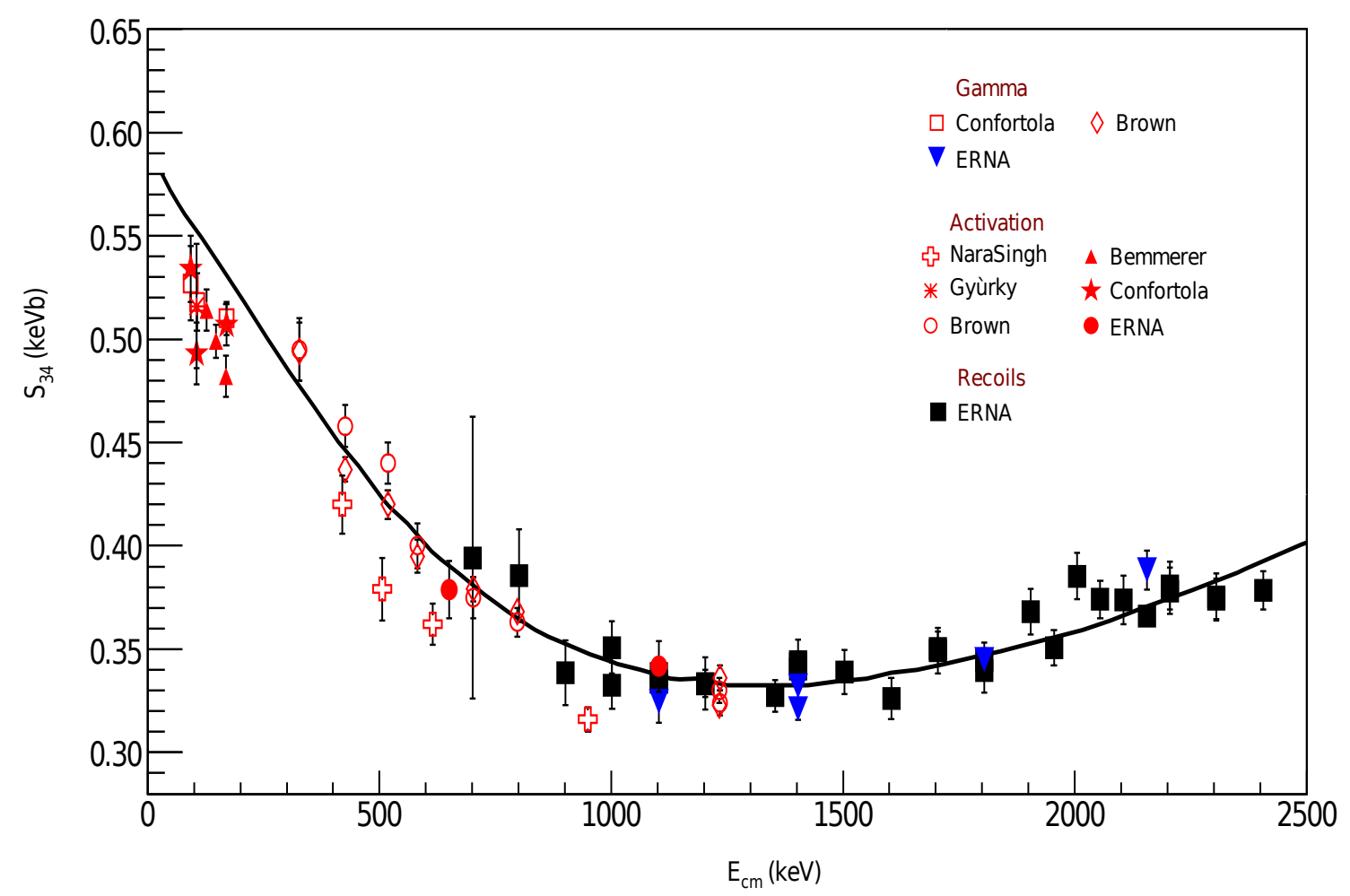

Figure 4: Comparison between S-factor theoretical calculation [22] and experimental data [23].

hazonov theoretical prediction [8]. Two new very preliminary data points are given at $80 \mathrm{keV}$ and $120 \mathrm{keV}$. Starting from the experimental data, a ${ }^{6} \mathrm{Li} / \mathrm{H}=(0.74 \pm 0.16) \times 10^{-14}$ abundance is obtained using the Smith, Kawano, and Malaney code [21].

\subsection{The ${ }^{3} \mathrm{He}(\alpha, \gamma)^{7} \mathrm{Be}$ reaction}

Not only ${ }^{6} \mathrm{Li}$ but also ${ }^{7} \mathrm{Li}$ was produced during the BBN. The leading reaction in the ${ }^{7} \mathrm{Li}$ production is the ${ }^{3} \mathrm{He}(\alpha, \gamma){ }^{7} \mathrm{Be}$, where the ${ }^{7} \mathrm{Be}$ decay in ${ }^{7} \mathrm{Li}$ through electron capture and consequent electron neutrino emission. The ${ }^{3} \mathrm{He}(\alpha, \gamma)^{7} \mathrm{Be}$ cross section has already been measured at LUNA but at energies lower than the one of interest for BBN where this cross section is important for solar astrophysics [11]. Unfortunately, the LUNA accelerator is not able to reach the BBN energy range. The measurement of this cross section at higher energies is thus part of the scientific program of LUNA-MV [10].

A new measurement of the ${ }^{3} \mathrm{He}(\alpha, \gamma){ }^{7} \mathrm{Be}$ cross section in the BBN energy range could connect the actual experimental data focused especially on high and low energy regimes, reducing possible systematics (figure 4).

Using the actual S-factor value, a ${ }^{7} \mathrm{Li} / \mathrm{H}=\left(4.89_{-0.39}^{+0.41}\right) \times 10^{-10}$ abundance is obtained [24].

\subsection{The ${ }^{2} \mathbf{H}(\mathbf{p}, \gamma)^{3} \mathrm{He}$ reaction}

The first nucleus produced in the Universe is deuteron. Its production fixes the beginning of BBN through the deuterium bottleneck. In this sense, primordial deuterium is fundamental in 


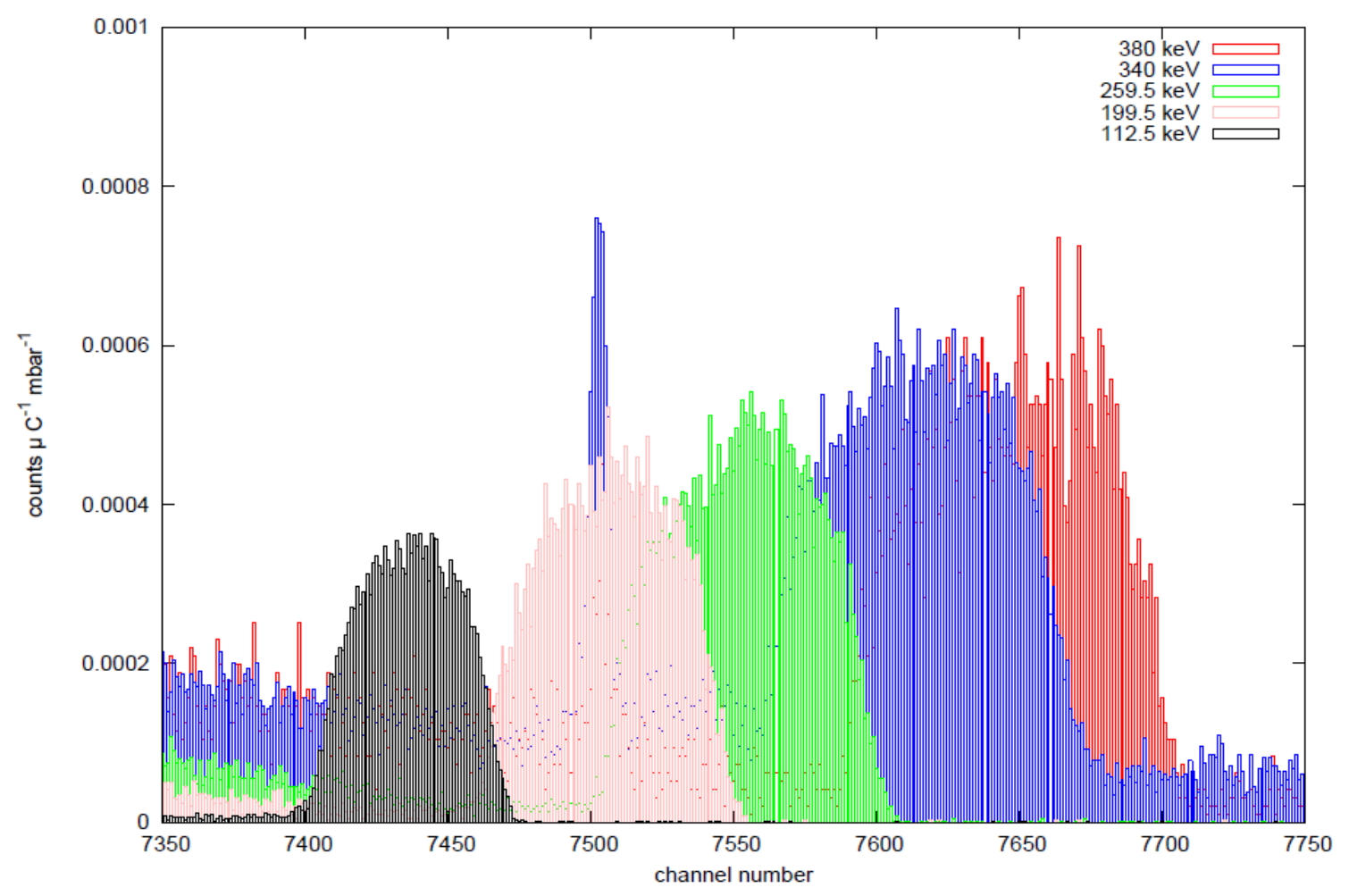

Figure 5: ${ }^{2} \mathrm{H}(\mathrm{p}, \gamma)^{3} \mathrm{He}$ signal for proton beam energies $\mathrm{E}_{p}=380 \mathrm{keV}, 340 \mathrm{keV}, 259.5 \mathrm{keV}, 199.5 \mathrm{keV}$ and $112.5 \mathrm{keV}$. The beam induced background is visible only when the beam energy approaches the $340 \mathrm{keV}$ ${ }^{19} \mathrm{~F}(\mathrm{p}, \alpha \gamma){ }^{16} \mathrm{O}$ resonance value.

cosmology and its abundance is strongly related to cosmological parameters such as, for example, the baryon density $\Omega_{b}$ and the effective number of neutrino families $N_{v}$ [25]. The main source of uncertainty in the primordial deuterium calculation comes from the ${ }^{2} \mathrm{H}(\mathrm{p}, \gamma)^{3} \mathrm{He}$ cross section [25]. Up to now, only a few authors measure this cross section in the BBN energy range with a precision at the level of 6-10\% [26]. As suggested by many authors [1,25,27], a new measurement campaign able to reach a $3 \%$ level precision is scheduled at LUNA. This will provide not only a direct measurement of the ${ }^{2} \mathrm{H}(\mathrm{p}, \gamma)^{3} \mathrm{He}$ total cross section but also the differential one putting constraints on ab-initio and $\chi$-EFT calculations. In order to investigate the possible systematics and backgrounds, a feasibility test has been performed at LUNA in October 2014. A windowless gas target filled with deuterium at 0.3 mbar pressure has been used. The reaction gammas have been consequently detected by a HPGe detector. No lead shielding and anti-radon box were provided. The ${ }^{2} \mathrm{H}(\mathrm{p}, \gamma)^{3} \mathrm{He}$ peak is shown in figure 5 .

A very preliminary analysis seems to show an increase in the cross section respect to the present literature data.

Two measurement campaigns are planned at LUNA in a close future. The former will measure the ${ }^{2} \mathrm{H}(\mathrm{p}, \gamma)^{3} \mathrm{He}$ total cross section at low-medium energies using an high efficiency BGO detector. The latter, instead, will cover the medium-high energies using a high resolution HPGe detector. Using this one, the ${ }^{2} \mathrm{H}(\mathrm{p}, \gamma)^{3} \mathrm{He}$ differential cross section is also obtained. Assuming the present 
data a ${ }^{2} \mathrm{H} / \mathrm{H}=(2.65 \pm 0.07) \times 10^{-5}$ abundance is obtained [25].

\section{Astronomical Observations}

The comparison between BBN calculations and astronomical observations is one of the most important evidence for the Big Bang $\Lambda \mathrm{CDM}$ model. Obviously not all the primordial nuclei could be detected due to their low abundances values and to the Universe chemical evolution. ${ }^{2} \mathrm{H}$ abundance can be estimated starting from the absorption lines of very old, and thus far, objects like high red-shifted quasars [27]. Due to systematics, the ${ }^{2} \mathrm{H}$ abundance value varies from author to author. Here we refer to the recent Pettini et al. analysis [28] that provide a ${ }^{2} \mathrm{H} / \mathrm{H}=(2.53 \pm 0.04) \times 10^{-5}$.

Excluding tritium, the second primordial nucleus produced during the $\mathrm{BBN}$ is the ${ }^{3} \mathrm{He}$. Observe its primordial abundance is however really hard given that, during their lives, stars produce and destroy ${ }^{3} \mathrm{He}$. Moreover, up to now we have only local observations that provide a ${ }^{3} \mathrm{He}$ abundance of ${ }^{3} \mathrm{He} / \mathrm{H}=(1.1 \pm 0.2) \times 10^{-5}$ [29]. Consequently the primordial ${ }^{3} \mathrm{He}$ abundance is not established yet [30].

Like for ${ }^{3} \mathrm{He}$ also ${ }^{4} \mathrm{He}$ is produced in stars, especially during their main sequence phase. In order to estimate the primordial ${ }^{4} \mathrm{He}$ is thus necessary to observe HII regions in old galaxies (compact blue dwarft galaxies) and extrapolate the detected ${ }^{4} \mathrm{He}$ abundance to the zero metallicity value [24]. Using a Markov Chain Monte Carlo technique, recently Aver et al. have determined the primordial helium abundance ${ }^{4} \mathrm{He} / \mathrm{H}=(0.2534 \pm 0.0083)$ [31].

Primordial Lithium is observed in stellar atmosphere of ancient low-metallicity stars located in our galaxy halo. Assuming that Lithium has not been depleted at the surface of these stars, the zero-metallicity abundance observed now correspond to the primordial one. Moreover, the good spectral resolution recently achieved allows to distinguish between the two isotopes ${ }^{6} \mathrm{Li}$ and ${ }^{7} \mathrm{Li}$ and consequently provide the two respective abundance values. The main contribution to Lithium absorption line comes from ${ }^{7} \mathrm{Li}$. The observed abundance is ${ }^{7} \mathrm{Li} / \mathrm{H}=\left(1.58_{-0.28}^{+0.35}\right) \times 10^{-10}$ [32]. The situation is almost unclear for what concerns the ${ }^{6} \mathrm{Li}$ detection. Recently, some authors [33] have pointed out that, by using three-dimentsional model atmospheres and dropping the assumption of Local Thermodynamic Equilibrium (3D NLTE model), many of the ${ }^{6} \mathrm{Li}$ detections may become insignificant. However, in some stars such as HD 84937, different 3D NTE models provides different results [34]. If a ${ }^{6} \mathrm{Li}$ detection is assumed, a $10^{-11}$ primordial abundance value should be considered [34].

\section{Conclusions}

In figure 6 the comparison between observed and calculated abundances is reported. The Helium astronomical data are in good agreement with the calculated one $\left({ }^{4} \mathrm{He} / \mathrm{H}=(0.2467 \pm\right.$ 0.0001) [1]) within error bars. A little tension is present for what concerns Deuterium. A larger number of $N_{v}$ and a consequent increase of the ${ }^{2} \mathrm{H}(\mathrm{p}, \gamma)^{3} \mathrm{He}$ cross section is desirable. This last condition is also requested in order to obtain a good agreement between experimental data and ab-initio calculations [35-37]. Finally a disagreement is present between the observed Lithium abundances and astronomical observations. The observed ${ }^{7} \mathrm{Li}$ is about three time less than the calculated one (the so called "First Lithium Problem") while the ${ }^{6} \mathrm{Li}$ abundance is three orders 
of magnitude higher ("Second Lithium Problem") [38]. Actually, Lithium abundances remain a brain-teasers for modern cosmologist but, at the same time, provide a unique possibility to search for possible new physics scenarios.



Figure 6: Comparison between observed (black horizontal bar) and calculated (blue curves) abundances versus the baryon density $\Omega_{b}$. The Planck value $\Omega_{b} h^{2}=0.02230 \pm 0.00014$ [1] is reported as a red vertical bar. The observed ${ }^{3} \mathrm{He}$ "local" abundance is shown as a green dashed bar.

Thanks to the good resolution achieved today in Nuclear Physics and Cosmology it's possible to predict primordial abundances of the light elements produced during the BBN era. Moreover, starting from the astronomical observations and cross section measurements, new limits on the cosmological parameters can be assumed. A new "Nuclear Cosmology" era is thus just started. 


\section{References}

[1] Planck Collaboration, P. A. R. Ade, N. Aghanim, M. Arnaud, M. Ashdown, J. Aumont, C. Baccigalupi, A. J. Banday, R. B. Barreiro, J. G. Bartlett, and et al. Planck 2015 results. XIII. Cosmological parameters. ArXiv e-prints, February 2015.

[2] G. Mangano, G. Miele, S. Pastor, and M. Peloso. A precision calculation of the effective number of cosmological neutrinos. Physics Letters B, 534:8 - 16, 2002.

[3] K. A. Olive and Particle Data Group. Review of Particle Physics. Chin. Phys. C, 38(9):090001, August 2014.

[4] Claus Rolfs and William Rodney. Cauldrons in the Cosmos. University of Chicago Press, Chicago, 1988.

[5] H. Costantini, A. Formicola, G. Imbriani, M. Junker, C. Rolfs, and F. Strieder. LUNA: a laboratory for underground nuclear astrophysics. Rep. Prog. Phys., 72(8):086301-+, August 2009.

[6] Carlo Broggini, Daniel Bemmerer, Alessandra Guglielmetti, and Roberto Menegazzo. LUNA: Nuclear Astrophysics Deep Underground. Annu. Rev. Nucl. Part. Sci., 60:53-73, 2010.

[7] A. Formicola, G. Imbriani, M. Junker, D. Bemmerer, R. Bonetti, C. Broggini, C. Casella, P. Corvisiero, H. Costantini, G. Gervino, C. Gustavino, A. Lemut, P. Prati, V. Roca, C. Rolfs, M. Romano, D. Schürmann, F. Strieder, F. Terrasi, H.-P. Trautvetter, and S. Zavatarelli. The LUNA II 400kV accelerator. Nucl. Inst. Meth. A, 507:609-616, July 2003.

[8] M. Anders, D. Trezzi, R. Menegazzo, M. Aliotta, A. Bellini, D. Bemmerer, C. Broggini, A. Caciolli, P. Corvisiero, H. Costantini, T. Davinson, Z. Elekes, M. Erhard, A. Formicola, Zs. Fülöp, G. Gervino, A. Guglielmetti, C. Gustavino, Gy. Gyürky, M. Junker, A. Lemut, M. Marta, C. Mazzocchi, P. Prati, C. Rossi Alvarez, D. A. Scott, E. Somorjai, O. Straniero, and T. Szücs. First Direct Measurement of the ${ }^{2} \mathrm{H}(\alpha, \gamma){ }^{6} \mathrm{Li}$ Cross Section at Big Bang Energies and the Primordial Lithium Problem. Phys. Rev. Lett., 113:042501, Jul 2014.

[9] C Gustavino. Bbn, neutrinos and nuclear astrophysics. Proceeding of Science [BORMIO], (050), 2014.

[10] Alessandra Guglielmetti. Nuclear astrophysics and underground accelerators. Physics of the Dark Universe, 4(0): 10 - 13, 2014. \{DARK\} \{TAUP2013\}.

[11] Heide Costantini et al. The ${ }^{3} \mathrm{He}(\alpha, \gamma){ }^{7} \mathrm{Be}$ cross section measurements at low energies. Nucl. Phys. A, 814:144-158, 2008.

[12] P D Serpico, S Esposito, F Iocco, G Mangano, G Miele, and O Pisanti. Nuclear reaction network for primordial nucleosynthesis: a detailed analysis of rates, uncertainties and light nuclei yields. J. Cosmol. Astropart. Phys., 2004(12):010, 2004.

[13] M. Anders, D. Trezzi, A. Bellini, M. Aliotta, D. Bemmerer, C. Broggini, A. Caciolli, H. Costantini, P. Corvisiero, T. Davinson, Z. Elekes, M. Erhard, A. Formicola, Z. Fülöp, G. Gervino,

A. Guglielmetti, C. Gustavino, G. Gyürky, M. Junker, A. Lemut, M. Marta, C. Mazzocchi, R. Menegazzo, P. Prati, C. Rossi Alvarez, D. Scott, E. Somorjai, O. Straniero, and T. Szücs. Neutron-induced background by an $\alpha$-beam incident on a deuterium gas target and its implications for the study of the ${ }^{2} \mathrm{H}(\alpha, \gamma){ }^{6} \mathrm{Li}$ reaction at LUNA. Eur. Phys. J. A, 49:28, February 2013.

[14] Michael Anders. S-factor measurement of the ${ }^{2} \mathrm{H}(\alpha, \gamma){ }^{6} \mathrm{Li}$ reaction at energies relevant for Big-Bang nucleosynthesis. Report HZDR-042 (2013) and PhD Thesis, Technical University of Dresden, 2013. 
[15] R. G. H. Robertson et al. Observation of the capture reaction ${ }^{2} \mathrm{H}(\alpha, \gamma){ }^{6} \mathrm{Li}$ and its role in production of ${ }^{6} \mathrm{Li}$ in the big bang. Phys. Rev. Lett., 47:1867-1870, December 1981.

[16] P. Mohr, V. Kölle, S. Wilmes, U. Atzrott, G. Staudt, J. W. Hammer, H. Krauss, and H. Oberhummer. Direct capture in the $3^{+}$resonance of ${ }^{2} \mathrm{H}(\alpha, \gamma){ }^{6} \mathrm{Li}$. Phys. Rev. C, 50:1543-1549, September 1994.

[17] J. Kiener, H. J. Gils, H. Rebel, S. Zagromski, G. Gsottschneider, N. Heide, H. Jelitto, J. Wentz, and G. Baur. Measurements of the Coulomb dissociation cross section of $156 \mathrm{MeV}^{6} \mathrm{Li}$ projectiles at extremely low relative fragment energies of astrophysical interest. Phys. Rev. C, 44:2195-2208, November 1991.

[18] F. E. Cecil, J. Yan, and C. S. Galovich. The reaction $\mathrm{d}(\alpha, \gamma)^{6} \mathrm{Li}$ at low energies and the primordial nucleosynthesis of ${ }^{6}$ Li. Phys. Rev. C, 53:1967-1970, April 1996.

[19] A. M. Mukhamedzhanov, Marco La Cognata, and V. Kroha. Astrophysical S factor for the ${ }^{15} \mathrm{~N}(\mathrm{p}, \gamma){ }^{16} \mathrm{O}$ reaction. Phys. Rev. C, 83:044604, 2011.

[20] F. Hammache, M. Heil, S. Typel, D. Galaviz, K. Sümmerer, A. Coc, F. Uhlig, F. Attallah, M. Caamano, D. Cortina, H. Geissel, M. Hellström, N. Iwasa, J. Kiener, P. Koczon, B. Kohlmeyer, P. Mohr, E. Schwab, K. Schwarz, F. Schümann, P. Senger, O. Sorlin, V. Tatischeff, J. P. Thibaud, E. Vangioni, A. Wagner, and W. Walus. High-energy breakup of ${ }^{6} \mathrm{Li}$ as a tool to study the Big Bang nucleosynthesis reaction ${ }^{2} \mathrm{H}(\alpha, \gamma){ }^{6} \mathrm{Li}$. Phys. Rev. C, 82:065803, December 2010.

[21] M. S. Smith, L. H. Kawano, and R. A. Malaney. Experimental, computational, and observational analysis of primordial nucleosynthesis. Astrophys. J. Suppl. Ser., 85:219-247, April 1993.

[22] K. M. Nollett. Radiative $\alpha$-capture cross sections from realistic nucleon-nucleon interactions and variational Monte Carlo wave functions. Phys. Rev. C, 63:054002, May 2001.

[23] A. Di Leva. Measurement of $3 \mathrm{He}(\alpha, \gamma) 7$ Be cross section with the recoil mass separator ERNA. PhD thesis, Ruhr-Universität Bochum, 2009.

[24] Alain Coc, Jean-Philippe Uzan, and Elisabeth Vangioni. Standard Big-Bang Nucleosynthesis after Planck. 2013.

[25] Eleonora Di Valentino, Carlo Gustavino, Julien Lesgourgues, Gianpiero Mangano, Alessandro Melchiorri, Gennaro Miele, and Ofelia Pisanti. Probing nuclear rates with planck and bicep2. Phys. Rev. D, 90:023543, Jul 2014.

[26] E.G. Adelberger, A. García, R. G. H. Robertson, K. A. Snover, A. B. Balantekin, K. Heeger, M. J. Ramsey-Musolf, D. Bemmerer, A. Junghans, C. A. Bertulani, J.-W. Chen, H. Costantini, P. Prati, M. Couder, E. Uberseder, M. Wiescher, R. Cyburt, B. Davids, S. J. Freedman, M. Gai, D. Gazit, L. Gialanella, G. Imbriani, U. Greife, M. Hass, W. C. Haxton, T. Itahashi, K. Kubodera, K. Langanke, D. Leitner, M. Leitner, P. Vetter, L. Winslow, L. E. Marcucci, T. Motobayashi, A. Mukhamedzhanov, R. E. Tribble, K. M. Nollett, F. M. Nunes, T.-S. Park, P. D. Parker, R. Schiavilla, E. C. Simpson, C. Spitaleri, F. Strieder, H.-P. Trautvetter, K. Suemmerer, and S. Typel. Solar fusion cross sections. II. The pp chain and CNO cycles. Rev. Mod. Phys., 83:195-246, 2011.

[27] Alain Coc, FaÃŕrouz Hammache, and JÃijrgen Kiener. Recent results in nuclear astrophysics. The European Physical Journal A, 51(3), 2015.

[28] Max Pettini and Ryan Cooke. A new, precise measurement of the primordial abundance of deuterium. Monthly Notices of the Royal Astronomical Society, 425(4):2477-2486, 2012.

[29] T. M. Bania, R. T. Rood, and D. S. Balser. The cosmological density of baryons from observations of ${ }^{3} \mathrm{He}^{+}$in the Milky Way. Nature, 415:54-57, January 2002. 
[30] Elisabeth Vangioni-Flam, Keith A. Olive, Brian D. Fields, and Michel Cass Ãl'. On the baryometric status of 3he. The Astrophysical Journal, 585(2):611, 2003.

[31] Erik Aver, Keith A. Olive, and Evan D. Skillman. An mcmc determination of the primordial helium abundance. Journal of Cosmology and Astroparticle Physics, 2012(04):004, 2012.

[32] A. Coc, J.-P. Uzan, and E. Vangioni. Standard big bang nucleosynthesis and primordial CNO abundances after Planck. J. Cosmol. Astropart. Phys., 10:050, October 2014.

[33] K. Lind, J. Melendez, M. Asplund, R. Collet, and Z. Magic. The lithium isotopic ratio in very metal-poor stars. Astron. Astrophys., 554:A96, June 2013.

[34] M. Steffen, R. Cayrel, E. Caffau, P. Bonifacio, H.-G. Ludwig, and M. Spite. ${ }^{6}$ Li detection in metal-poor stars: can 3D model atmospheres solve the second lithium problem? Mem. S.A.It., 22:152, 2012.

[35] M. Viviani, A. Kievsky, L. E. Marcucci, S. Rosati, and R. Schiavilla. Photodisintegration and electrodisintegration of ${ }^{3} \mathrm{He}$ at threshold and pd radiative capture. Phys. Rev. C, 61:064001, Apr 2000.

[36] L.E. Marcucci, Kenneth M. Nollett, R. Schiavilla, and R.B. Wiringa. Modern theories of low-energy astrophysical reactions. Nuclear Physics A, 777(0):111 - 136, 2006. Special Isseu on Nuclear Astrophysics.

[37] L. E. Marcucci, M. Viviani, R. Schiavilla, A. Kievsky, and S. Rosati. Electromagnetic structure of $a=2$ and 3 nuclei and the nuclear current operator. Phys. Rev. C, 72:014001, Jul 2005.

[38] B. D. Fields. The Primordial Lithium Problem. Annu. Rev. Nucl. Part. Sci., 61:47-68, November 2011. 